\title{
A Conceptual Paradigm For Internet And Search Engine Marketing
}

\author{
Alyssa Ashley, University of Baltimore, USA \\ Michael V. Laric, University of Baltimore, USA \\ Peter M. Lynagh, University of Baltimore, USA \\ Craig A. Vollmer, University of Baltimore, USA
}

\begin{abstract}
The Internet continues to affect the relationships between business and their customers, and companies must adjust their online marketing strategy, if they want to increase revenues and market share, respectively. The emergence of the Internet over the last decade has had a major impact on the communication industry. The Internet is constantly evolving and is continually undergoing a rapid evolutionary process in both capabilities and uses. Marketing managers have begun to direct their focus and resources towards the Internet in an attempt to capture and garner new consumers. The Internet has forever changed the customer purchasing experience, and companies must alter their online marketing strategy, if they want to increase revenues and market share, respectively.
\end{abstract}

\section{INTRODUCTION}

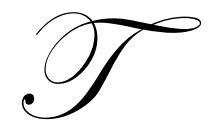

his paper proposes a conceptual paradigm for Search Engine Marketing (SEM,) an integral part of today's Internet marketing strategy. SEM focuses on both organic search engine optimization (SEO) and pay per click (PPC) advertising. Search engines help-users locate information on topics of interest. A number of search engine strategies have been developed. Some are available as a feature of a Web site that provides many services (e.g., AOL.com), while others are the main feature of a Web site and the reason users come to the site. The main two search engines are Google which also drives a few others like AOL, MySpace, Netscape, and CompuServe, and Yahoo! which also drives a few others like AltaVista, AlltheWeb, and GoodSearch.

Web search engines work by first searching through, then storing, information about myriad Web pages which they have retrieved. The process is facilitated by programs known as crawlers or spiders which follow links. The pages are then analyzed and indexed using words extracted from titles, headings, and special fields. The index data are stored in a database which is used by searchers looking for information. When a user enters search words or phrases, the engine looks up the index and provides a listing of Web pages that best match the searchers input data. The summary contains the Web document's title and sometimes parts of the text. Experienced users can use Boolean terms (AND, OR and NOT) to refine their search queries.

While there may be thousands of Web pages returned by the search engine, some may be more relevant to the user than others. Often the user will not look beyond the results presented on the first page. As noted earlier, search engines vary widely in methods used to provide results, and these also vary over time as Internet usage changes and new techniques evolve. As more search engines seek advertising revenues, some employ the controversial practice of allowing advertisers to pay money to have their listings ranked higher in search results.

SEO is the practice of using a range of techniques, including augmenting HTML code, Web page copy editing, site navigation, linking campaigns and more, in order to improve how well a site or page gets listed in search engines for particular search topics (SEMPO, 2005, 3). PPC advertising is another marketing tactic used in search engine marketing to take the consumer by storm. PPC which is also associated with other names such as "cost-per-click" and "paid search" has quickly moved up the ranks and is considered a cornerstone within the model known as pull advertising. 
In addition to a historical perspective of organic SEO versus PPC advertising depicting the various successes and failures of each, a paradigm is developed which stresses the efficacy of utilizing a combination of both SEO and PPC strategies. It recommends that marketing managers make decisions about their overall SEM strategy by combining both strategies.

\section{The Paradigm}

The paradigm looks at SEM holistically and from a longer term perspective. It offers a conceptual approach that will assist marketing managers develop an SEM strategy. The paradigm (shown in Exhibit 1) focuses on the assumptions used to inform marketing decisions makers regarding the validation of their SEM strategy.

Web search engines (such as Google and Yahoo!) are depicted at the top of the diagram, above a fountain with three funnels. The search results cascade down to the funnels (PPC and SEO). When consumers start a search they get back results in the form of links to Websites being funneled down. This is portrayed as water in the paradigm's fountain allegory. Like a water pump pushing the water up to the top of the fountain, information flows back to the consumer and the digital flow traffic gets filtered back down into the search engines and continues to move in a circular fashion.

In a fountain, water trickles down from one tier to the next; rain, however could fall into the either fountain level directly. In that sense a consumer can gain access to a desired Website by traveling directly to the site directly from the Web. Consumer traffic can also be funneled through the Web and or the search engines each of which might have a slightly different pattern. The paradigm arrows point both ways suggesting that consumer traffic is being funneled through PPC or through SEO to the. Not all SEM users will be converted into a customer on a given site. They may return back to the Web and begin to search again. It can also be likened to water evaporating from the fountain and moving back up to the 'cloud' of the search engine marketing.

The paradigm focuses on informing marketing decision makers regarding the validation of their SEM strategy. They can target the Web search engines such (Google and Yahoo!) whose engines return information which cascades down to either or both PPC and SEO, providing information and link to the customer who is ultimately clicking on the links resulting in the information being funneled down into various Websites, and hopefully to ones for which the marketing managers are trying to increase traffic and sales.

The emergence of the Internet over the last decade has been as one industry observer notes: "The biggest innovation in the advertising industry during the last 70 years" (Steele, 2007). It very simply has made the world a whole lot smaller by facilitating commerce, communicating with family and friends, and storing content in an easily searchable format. The Internet is still evolving and is constantly undergoing an evolutionary process in both capabilities and uses.

In this new world of Internet marketing, SEO and PPC are considered to be complementary strategies that ultimately enhance the customer online experience. Therefore, the ideal SEM strategy should consider both tactics in a mix to maximize sales, revenues and metrics as part of a long-term initiative. As validation for the methodology, Jim Collins advocates a long-term strategy much like building a clock (i.e. long-term strategy), rather than simply telling time (short-term strategy) (Collins, 2002; p. 22). Hence, his belief is that the true and enduring corporations tend not to take a short-term perspective, but instead holistically approach their business strategy with the rigor that stands the test of time, regardless of the prevailing economic or business conditions. In example after example, Collins illustrates Wal-Mart versus Ames, Motorola versus Zenith, and Walt Disney versus Columbia Pictures proving that each company had equal opportunity to embrace an "architect" or "clock builder" to preserve the core company ideology and stimulate progress. The Internet marketing strategy is no different. Companies like Google, Yahoo, and Alta Vista are wonderful examples of those who take a long-term approach to building their search engine business models and have done so since the advent of the Web. 


\section{INTERNET MARKETING AND SEARCH ENGINES}

\section{Internet Marketing (IM)}

The Internet appeared on the scene more than three decades ago during the Cold War - sponsored by the Department of Defense Advanced Research Projects Agency known to many by its acronym, DARPA. Through the 1980 's it remained the domain of military and academic users who were willing to master the requisite “computerese." It was text based. By the middle of the 1980's, the military part was split off, but the commercial use on the Internet was still funded by the government.

All that changed with the Web - a user interface which allowed "point and click" access and incorporated graphics, sound and video. This ushered a period of rapid development of tools and applications which facilitate information acquisition and exchange by non-technical users. The first browser appeared on the scene in the mid 1990's and dramatically increased users ability to view Web documents that incorporated the Web's multimedia authoring tools. As these tools continue to evolve, they provide a means to creating an Internet presence for any business - large or small, as well as new modes of buying, selling, and competition, to name a few.

In a world of just over 6.5 billion people, the Internet has played a major role in the transformation of the world economy. This is especially relevant in the realm of marketing. Media such as broadcast and print are being supplanted by digital communications. The Web enables advertisers to have an interactive medium for reaching a savvy, technologically educated target market, while also creating a medium that has led advertisers to rethink traditional marketing efforts with respect to new approaches in advertising (Shiva, 1997; p. 3). This has resulted in the "recent shift in advertising/marketing dollars into online channels that deliver less expensive distribution, greater personalization, and higher response and tracking rates than traditional marketing dollars are able to capture." (Boswell, 2002, p. 1)

In the early stages of IM many businesses tried to develop Web sites. The upsurge of interest in the Internet during the 1990s was directly related to the growth in business Websites focused on creating graphics, audio, and video - multimedia which was eye catching and attractive to the browsing public. Users could use one of the browsers (Microsoft Internet Explorer, Fire Fox or Netscape Navigator) to "shop" among and within businesses. Equally important from a marketing perspective, companies could gather data which could be used for marketing as well as for gaining feedback about products or services

This interactive aspect of the Web is one of its essential features and potentially the most important reason for the increased use of the Web in business strategy. IM is a two way street allowing interactive communications with customers thereby helping to create good customer relationship. Keeping close to customers and the marketplace is another important goal for IM providing many opportunities for doing so.

\section{Search Engines And Search Engine Marketing (SEM)}

Search engines help-users locate information on topics of interest. A number of these have been developed. Some are available as a feature of a Web site that provides other services (e.g., AOL.com), while others are the main feature of a Web site and the reason users come to the site. The main two search engines are Google (http://www.google.com) which also drives a few others like AOL, MySpace, Netscape, and CompuServe, and Yahoo! (http://www.yahoo.com) which also drives a few others like AltaVista, AlltheWeb, and GoodSearch.

The basic underlying assumption of SEM is that the potential target markets are using key words and phrases when using search engines to learn about products and services they intend to purchase. This is the basic logic underlying some of the search engine principles. Since most search engines use many criteria to determine relevancy of Web pages (in addition to the rank order) marketers must increasingly become familiar with the logic and operations of search engines. Search engines have become increasingly more popular as large amounts of useful information are stored on the Internet. They allow users to ask for specific information, under defined criteria, by 
entering a given word or a phrase. The result is displayed providing a list of references that match the word or phrase.

Web search engines work by first searching through, then storing, information about myriad Web pages which they have retrieved.. The process is facilitated by programs known as crawlers or spiders which follow links. The pages are then analyzed and indexed using words extracted from titles, headings, and special fields. The index data are stored in a database which is used by searchers looking for information. When a user enters search words or phrases, the engine looks up the index and provides a listing of Web pages that best match the searchers input data. The summary contains the Web document's title and sometimes parts of the text. Experienced users can use Boolean terms (AND, OR and NOT) to refine their search queries.

While there may be thousands of Web pages returned by a search engine, some may be more relevant to the user than others. Sometimes users will not look beyond the top results presented on the first page. As noted earlier, search engines vary widely in the methods used to provide results, and these also vary over time as Internet usage changes and new techniques evolve. As more search engines seek advertising revenues, some employ a controversial practice of allowing advertisers to pay money to have their listings ranked higher in search results. Whether referring to business-to-business, (B2B) business to consumer (B2C) or peer to peer, also known as consumer-to-consumer - C2C) e-commerce, everyone wants to have their very own piece of the global marketplace

\section{SEARCH ENGINE MARKETING (SEM) ELEMENTS -SEO AND PPC}

IM, also known as digital marketing, is a more finely tuned, unobtrusive, strategy to providing the consumer with a truly customized and unique experience. IM is concentrated more on providing potential clients and consumers with information that is both desired and relevant in order to assist in the decision making, purchasing or investing process. SEO, PPC and permission-based email notification such as bulletins, newsletters, and promotion coupons are just a few of the IM options available. The first two, SEO and PPC are primary drivers of any SEM strategy, which according to the Search Engine Marketing Professional Organization (SEMPO) is the entire set of techniques and strategies used to direct more visitors from search engines to marketing Web sites.

\section{Search Engine Optimization (SEO)}

SEO is the practice of using a range of techniques, including augmenting Hyper Text Markup Language (HTML) code, Web page copy editing, site navigation, linking campaigns and more, in order to improve how well a site or page gets listed in search engines for particular search topics (The State of Search Engine Marketing 2005; 3). The dimensions of search engine optimization have both positive and negative implications. On the positive side, SEO allows companies to forge "sticky" customer relationships, leverage the pull versus push medium dynamic and build brand awareness. Everyday businesses are looking for new customers, and the internet is the premier venue to capture new prospects; therefore, one way to build good customer relationships is to have better content than your competitors. Karen Bannan (2004) offers the following tips to increase your site search engine placement:

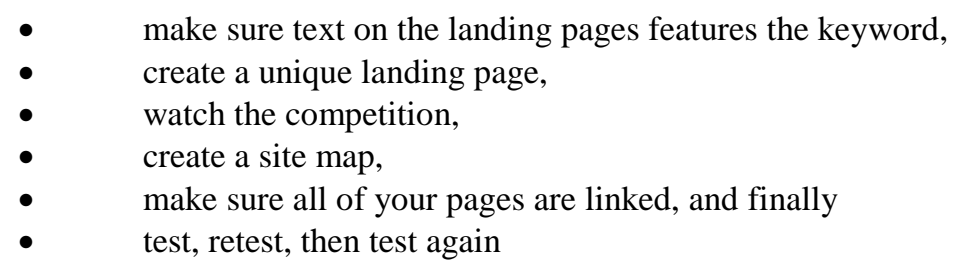

Metrics used when attempting to measure "stickiness" include: how often the same people visit a site, how long they stay on the site and where they go in the site or when they leave? In turn, this drives the idea that the content available must take into consideration the end-user instead of some search engine algorithm. Another benefit to search engine optimization is capitalizing on the pull-medium dynamic. In today's world customers want a personalized shopping experience, subsequently, it is important to understand customer behaviors and preferences in determining their consideration set. John Deighton (Bloomberg, 2007) identified the following characteristics: 
- the customer initiates contact,

- the customer is seeking information (pull),

- $\quad$ it is a high intensity medium,

- a company can gather and store the response of the individual and

- customers can download and store information

SEO provides another benefit in allowing one to promote and build one's brand. Assuming Web content is regularly being refreshed and updated; users will add it to their favorites and thereby bring it to "top of mind" status. For instance, Web MD, a source of medical information can be personalized, creating a niche brand that users will come to appreciate. (http://www.Webmd.com/)

On the other hand, search engine optimization has its challenges in the terms of style versus substance and potentially decreased Web traffic and revenue. The debate surrounding style versus substance raises the issue of should the Website structure appeal to search engines or should the Websites attempt to have the consumer in mind when designing the Website. A case for the latter can be made because there have been several examples of companies who attempted to play the system. For example in 2006, Ricoh and BMW were both "blacklisted" from Google's search for trying to manipulate search engine results; as a result, these two companies lost valuable traffic and revenue opportunities (Kerwin and Taylor, 2006). According to Google, both companies engaged in unethical search-engine optimization technique called "doorway;" pages crammed with hidden keywords that misrepresent a Web page's content, or JavaScript redirection that shuttled users to a different URL than the search identified (Kerwin and Taylor 2006;1). Fortunately for both companies they have since addressed those issues and resubmitted their content to Google. Additionally, Anthony Muller, who is a director of search optimization at Range Online Media, suggests companies should avoid the following SEO illegal tactics: shadow domains, doorway pages, invisible text, link farms, and cloaking (Search Optimization Tactics to Avoid, 2002, p. 1-2).

\section{Pay Per Click (PPC)}

PPC advertising is another marketing tactic used in search engine marketing. PPC which is known by other names including cost-per-click and paid search has quickly moved up the ranks and is considered a cornerstone within the model known as pull based advertising (Guarnaccia, 2007). PPC was started by Yahoo!'s Search Marketing Solutions (formerly known as Overture) in 1998. Google has since become the leader in the industry by securing over half (nearly 55\%) of the global search engine market share. The increasing gap in market share between Google and Yahoo! (number one and two respectively) is a concern for business owners. As the distance between the leaders increases, the cost of participating in PPC is rising rapidly (Raychaudhuri, 2007).

PPC, is the quickest and easiest way to display a business' Website on page one of a given search engine. PPC uses an auction-based model in which a business and its associated content are sequenced on their Website. They bid on the price they are willing to pay for every potential customer who clicks on their Website as a result of typing in that keyword into the search engine. Many businesses will be retrieved as a result of the search which is invoked when the customer types in a particular keyword. The businesses gain a specific rank on the search result based on their bid price. The cost per keyword ranges from as little as one penny to $\$ 50$, based on how likely they are to result in a successful search. Some will pay as much as $\$ 50$ per click for keywords so as to maintain the number one ranked position. Since the bidding process is available around the clock, bidding the most one day does not guarantee placement the next day - someone else may bid the up the price.

PPC advertising offers advantages over the more traditional forms of search engine marketing. All of which can be summed up with two words, quick and easy. PPC was designed to provide a very quick and painless process to begin advertising on search engines.

There are problems associated with PPC. An informed consumer knows how to recognize the difference between a PPC listing which can simply be considered an ad, and a genuine site that was found through the search engine. Many informed consumers often disregard PPCs focusing instead on the search results, a result of SEO rather than PPC. A second consideration and perhaps more devastating for the budget of the company, is the threat 
of click fraud from using a PPC. There are two main types that currently are causing major problems. The first is known as competitor click fraud. This occurs when a company intentionally clicks on one of its competitor's PPC link repeatedly in order to drive up the cost to them of a click through. The second type is known as network click fraud. Network partners who establish PPC links for clients receive a portion of the click through rate every time someone clicks on the link. Because of this type of compensation, these network partners have found various ways to repeatedly click on a client's link in order to receive more money themselves. Although network partners tend to be savvier than a disgruntled competitor, both of these types of click fraud can be detrimental.

\section{THE SEM CONCEPTUAL PARADIGM}

In looking at SEM holistically and from a longer term perspective, there is a need for a conceptual paradigm that will assist marketing managers develop an SEM strategy. The proposed paradigm focuses on the assumptions used to inform marketing decision makers regarding the validation of their SEM strategy.

Certain assumptions help in presenting the paradigm which is shown in Exhibit 1. Web search engines (such as Google and Yahoo!) are at the top, above the three funnels which are depicted as a fountain. The search results cascade down to PPC, SEO; when consumers start a search they get back results in the form of links to Websites being funneled down. This is portrayed as water in the paradigm's fountain allegory. Like a water pump pushing the water back up to the top of the fountain, information flows back to the consumer and the digital flow traffic gets filtered back down into the search engines and continues to move in a circular fashion.

In a fountain, water trickles down from one tier to the next; rain, however, could fall into the lower fountain level directly. In that sense a consumer can gain access to a desired Website using one of several links or traveling directly to the site from the Web. Consumer traffic can be funneled through the Web and or the search engines each of which might have a slightly different pattern. The arrows pointing both ways suggest that consumer traffic goes both ways. Information is being funneled through PPC or through SEO. Not all users will be converted into a customer on a given site, or a given visit. They may return back to the Web and begin to search again. It can also be likened to water evaporating from the fountain and moving back up to the 'cloud' of the search engine marketing

In looking at SEM holistically from a long term perspective, the focus is on the goal of informing marketing decision makers regarding the validation of their SEM strategy. At the top, above the fountain are the Web search engines such as Google and Yahoo! whose engines return information which cascades down to PPC, SEO, providing information and links to the customer who if ultimately clicking on the links will resulting in the water or information being funneled down into various Websites.

Like a water pump pushing the water back up, consumer traffic gets filtered back into the search engines. The process is interactive and continues to move in a circular fashion. Although the visual of the fountain serves as a good starting point, further explanation is required. Unlike a fountain where water trickles down from one tier to the next, consumer traffic being funneled through the Web and search engines has a slightly different pattern. For example the customer may go directly to a Website without utilizing the search engine. That is the purpose of bookmarks and cookies. That being said, the main SEM effort is directed at search outcomes. As the arrows above suggest, a consumer can gain access to a desired Website using one of the following: traveling directly to the site from the Web, being funneled through PPC to the site or being funneled through SEO to the site. Furthermore, these arrows show that consumer traffic goes both ways. Not all users will be converted into a customer on a given site. They may return back to the Web and begin to search again. 


\section{Exhibit 1: The SEM Conceptual Paradigm}

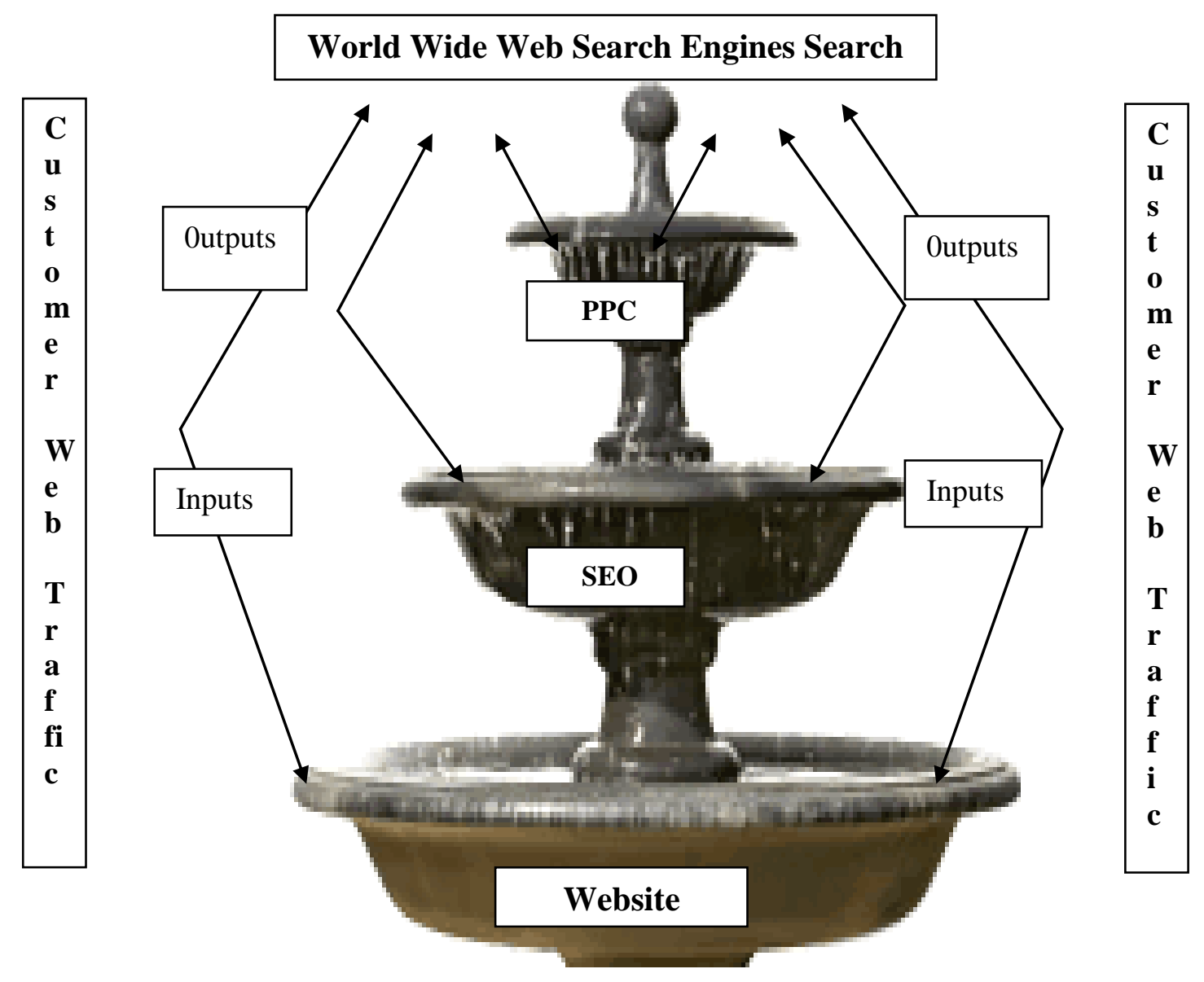

\section{Paradigm's Prerequisites and Assumptions}

There are of course prerequisites to the process described in the paradigm. The first one is that the customer has access to a computer and that this computer has access to the internet. Furthermore, customers must be computer literate and internet savvy. This may sound very elementary but the consumer must also become acclimated to the search engine universe. There are instances in which search results generate unwanted information. It would be better if search engines could have the knowledge and ability to differentiate between what searchers are looking for and what is junk. This could significantly contribute to the overall Internet experience, and through that affect the efficacy of the SEM strategy.

Some of the pitfalls that may be encountered when using search engines e.g., shadow domains, doorway pages, invisible text, link farms, and cloaking often lead to a frustrating and overall poor experience with search engine interaction and can lead to the deterioration of the overall Internet experience which could impact a consumer's purchase decision.

The various stages of the consumer's purchasing continuum are another factor that must be considered. There are several positions along the continuum. First, there are those consumers who are uninformed/unbiased in their buying decision; not satisfied with an existing product, and are looking for a replacement; made the purchase and are doing continuous research/validation purchase decision to minimize cognitive dissonance. The early 
purchase decision cycle would consist of the following characteristics: uninformed, unbiased, and unaided in brand awareness. For example, if consumers were in the market for a new car, they would use search engines to find detailed and relevant research regarding the car. They would discover sites such as Edmonds, which can be found at (http://www.edmunds.com/), Consumer Reports, site which can be found at (http://www.consumerreports.org/cro/index.htm) and Road and Track magazine, at (http://www.roadandtrack.com/) just to name a few. As a result of this information and data collected, the buyer becomes informed regarding the various product attributes.

In the second customer segment, the consumers who currently have a product or service, but are wavering in their loyalty to the current provider, are at a "tipping point" for a new provider or product. The first segment of disloyal consumers is identified as a detractor by Fred Reichheld in his book entitled "The Ultimate Question: Driving Good Profits and True Growth" (2006) defines detractors as those who rank on a satisfaction loyalty scale of one through six out of a ten. Conversely, he states that passive consumers, the second segment of potential disloyal consumers, are those who rank as a seven or eight out of ten. These two segments make up potential consumers who would be utilizing this model for a potential new provider.

In the third consumer segment, the customers are assumed to have an ongoing thirst for validating their existing purchase decisions. They do that by conducting additional researches through the use of the Internet and search engines, thus eliminating buyer's remorse, if the right results (Websites) are retuned by the search engines.

Finally, the last dynamic of the paradigm takes into account consumers who are looking to maximize value for their dollar (i.e. in terms of quality, price, and authenticity). Increasingly, customers use search engines to maximize their return. For example, the eBay community allows buyers and sellers to come together in an online marketplace where price is the first differentiator when looking at a product. However the buyer has little assurance other than the reputation of the seller that the merchandise is genuine.

\section{Input Aspects Of Brand Building Using SEM Strategies}

Building a brand is in direct correlation with and responsible for cultivating credible and durable relationships with customers. An integral part of business is building sticky relationships that are profitable, satisfying and long lasting for both parties. Regardless of whether a customer accesses a site using PPC or SEO, creating an organically rich Website improves the success rate of establishing a credible relationship with consumers. Maintaining satisfied customers will in turn breed customer loyalty.

The second characteristic for input strategies requires a deeper understanding of the push/pull dynamic outside of the traditional integrated marking communication strategy. Advertising on the internet is more fragmented than traditional media, and is more customized. A combination of PPC and SEO offers consumers a pull form of marketing that enables them to develop their own sense of ownership in developing decision criteria. As a result, this dynamic influences the paradigm because marketers must consider more carefully how to reach their intended targeted audience.

The third input involves the fact that any Website will not be ranked high by the search engine algorithms if the content is not relevant, organized, monitored and updated. This involves creative thinking and a long term orientation into how Websites are constructed and maintained. Whalen (2007) notes that "Simply being found in the engines isn't always enough.

Another input consideration is that marketers must evaluate their overall URL strategy and tactics. In particular, marketers should consider whether or not they wish to employ a vanity URL and alternative 'failsafe' URL's in order to drive and capture traffic that would otherwise be unable to navigate to their site. Vanity URL's are typically a shorter version of a company's name used in order to reduce inaccuracy in attempting to locate a site.

Another consideration when developing a content rich Website is factoring in the number of words used in order to attract search engines and ultimately customers and end users. This is a very important step shared by both 
SEO and PPC; however the steps associated with each form of search engine marketing vary. From an organic search engine perspective content taxonomy should be based on providing the end user with useful and actionable information.

In PPC, on the other hand, a word list is created based upon careful consideration of a site's customers' thought pattern. In PPC where words are bid on and paid for based upon click through rate, a site must first develop a keyword list based on keyword research that would target specific key phrases a potential consumer may enter on a search engine. These words may range from the extremely popular to the seldom used; however, keywords that under perform do not cost extra for a site to maintain, yet words that are not bid upon can cost a site dearly by potential loss in traffic. For both forms of search engine marketing, the monitoring of content and keywords is an ongoing process that should not and cannot be overlooked. By monitoring Website referrer logs, the paradigm can begin to pick up surprising patterns and trends as to how customers are searching the internet allowing for adjustments that will over time seamlessly fit a customer's preferences.

\section{Output Aspects Of Brand Building Using SEM Strategies}

The clock building approach takes into account the inputs and the associated outputs which yields a robust search engine paradigm enabling key stake owners to make informed decisions. All the outputs are interdependent with the inputs. These outputs include: metrics, increased loyalty and satisfaction and high search engine ratings.

This paradigm takes into account both quantitative and qualitative factors that ultimately measure the successes and/or failures through the use of metrics. Acquiring and analyzing these metrics, as well as harnessing the knowledge gained from analysis is directly and indirectly affected by the successful implementation of several of the inputs mentioned above including: understanding the push/push dynamic, building the brand and organizing/continually updating a site's content. One of the problems is how to depict success in search engine strategy. Metrics as a collective group represent key indicators, which are used to measure and quantify key accomplishments such as: traffic, conversion rate, click through rate, return on investment, cost per click and total number of online sales.

The second output in assessing customer loyalty and satisfaction is a result of multiple inputs including: establishing credible and durable relationships and cultivating the brand. This can be successfully achieved through implementing market research questionnaires as a way to nurture and deepen the relationship between buyer and seller. Meantime the ultimate goal is to determine how sticky the relationship is that ultimately drives customer lifetime value (LTV).

When looking at SEO and PPC, it is important to assess how well the sellers are building such long term relationships. This goal is to cultivate and increase customer lifetime value which is the "Holy Grail" measure of success. Otherwise in the case of PPC, acquisition costs for customers will outweigh actual benefits if marketers cannot cross sell future products and services to create durable customer relationships.

Maintaining high search engine rankings via first page results on Google, Yahoo!, etc., can be achieved through utilizing the inputs of organized, timely content and monitoring the community of words. The ultimate goal is to always achieve first page search engine results. It is important to note that it is unreasonable for any consultant or firm to be able to guarantee a consistent number one ranking. According to Google, "No one can guarantee a number one ranking... Beware of SEOs that claim to guarantee rankings, allege a 'special relationship' with Google, or advertise to 'priority submit' to Google" (Web Master Guidelines, 2007). Peters (2007) found that "just 20\% of users scroll down far enough to see the $10^{\text {th }}$ place match, let alone click on it." Therefore, in order for a site to achieve "above the fold" status consistently, monitoring and updating content is a necessity. The use of PPC strategy tactics in conjunction with organic site rankings enhances the opportunities for a site to not only be noticed, but to be clicked on as well. Lyubovitzky, claims, “...if your site comes up on the first two pages on major organic search and there's a link to a paid search, it gives the user more confidence. That visitor may feel more comfortable and have a higher level of trust" (Bannan, 2005). 
Exhibit 2, entitled: "At Your Service," re-emphasizes how imperative first page placement is for a site. In turn, this underscores the importance of integrity within a site. According to Google, "Their complex, automated methods make human tampering with their results extremely difficult. The company's goal is to provide an easy, honest and objective way to find high-quality Websites with information relevant to the user's search." (Web Master Guidelines 2007) There are no shortcuts to building an insightful and content-friendly resource for current and potential users.

Currently, a significant limitation of search engine rankings is the impersonal nature of the results. Regardless of a user's background or history, anyone who types in the specific keyword will receive the same results. However, the future trend in search engine marketing is a personalization of search results based on a user's Web history and profile. This contributes to a more user friendly and customized experience for the customer which validates the pull dynamic. Marketers are progressing towards the concepts of behavioral generational and neuromarketing with the end user in mind as an emerging and growing trend that transcends all business paradigms and industries engaging in search engine marketing.

\section{TESTING OF THE SEM STRATEGY INDUSTRY PRACTICES}

\section{National Fast Food Chain (NFF)}

NFF has had a Website set up for over six years. Initially it was designed to simply inform both customers and investors about the company and what it stood for. However, the site quickly began to mature as NFF noticed that people were trying to locate store information for ordering food on their site. Between 4 and 5 years ago, the site was upgraded to be able to collect email addresses for the use of informing customers about NFF's various promotions. To show how this service has grown, in 2004 NFF had over 150,000 unique email addresses, in 2007 there are over 500,000. The Website continues to grow and mature. Online ordering (OLO), launched in the middle of 2006, has become a primary focus for the company's growth.

NFF has used both SEO and PPC; however there is much more of a push towards PPC because of how NFF maintains its site. In 2006, the company invested \$200,000 in SEO to enhance the site; at this point there is no intention of investing much more. NFF ranks high (top three) in organic search because of their strong brand presence. The company is more concerned with having a Website that is on the cutting edge and engaging rather than making it $100 \%$ optimized. The formats of many of NFF micro sites that drive customers to interact with the NFF brand aren't recognized organically. Therefore, the company views PPC as the way to go. Ideally, the goal is to purchase over 1,000 words that are NFF (and its product) specific for the entire country. It is anticipated that there will certainly be a plateau in PPC, NFF feels that PPC is an important player in reaching their goal. 


\section{Exhibit 2: At Your Service}

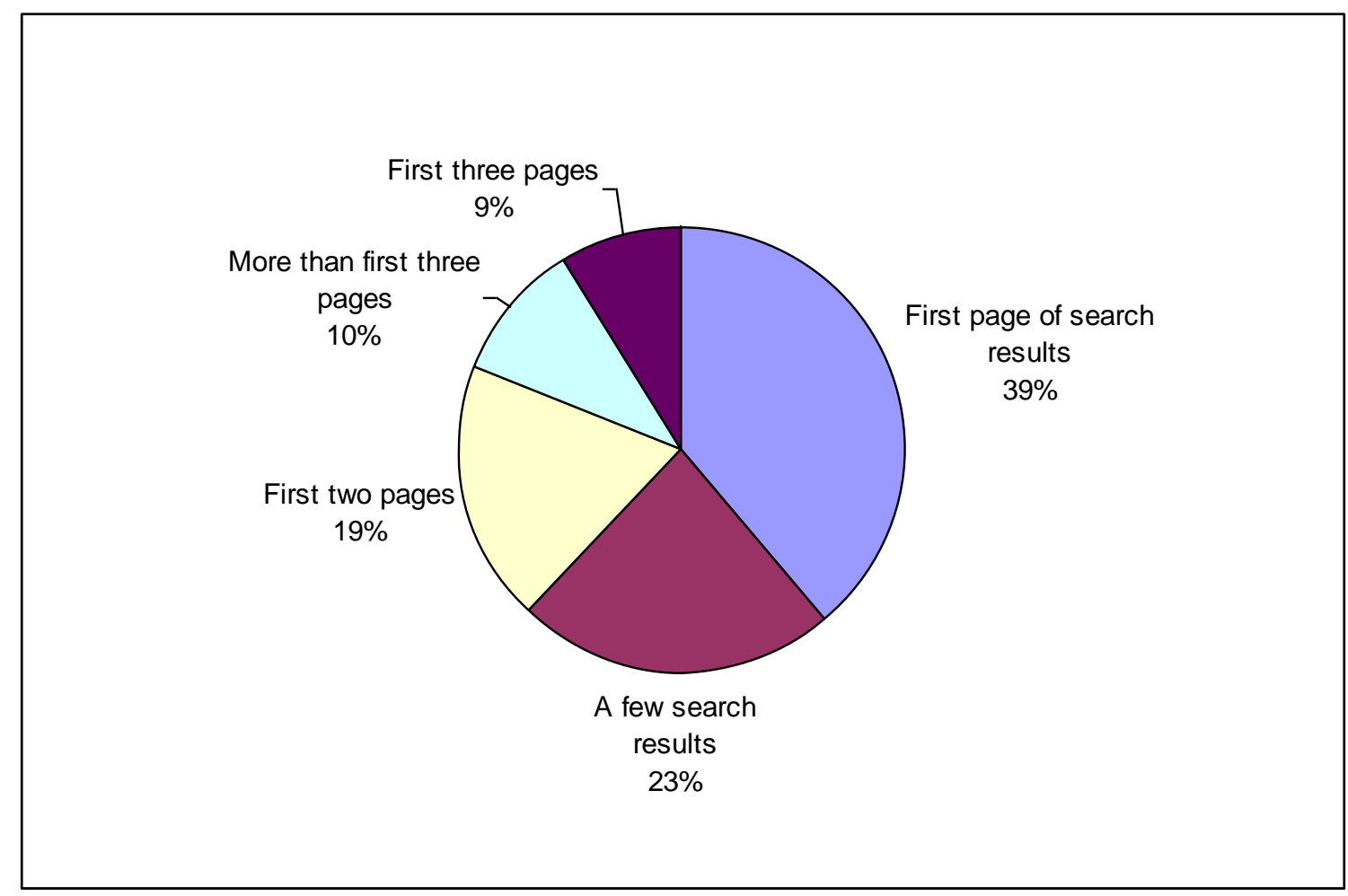

Sources: JupiterResearch and IProspect

\section{Financial Services Company (FSC)}

FSC has been in the PPC environment since 2000, and its progression through the years has been steady, slow, and successful. FSC has a corporate culture that tends to be conservative and cautious in nature. Therefore, when the Internet began emerging as a new technology for marketers, the firm initially made a $\$ 100,000$ investment and was managing a 500 keyword list. While the initial amount was certainly not significant in comparison to its main direct competitors, it signaled a strategic shift for FSC which had in the past allotted its entire advertising budget to traditional media outlets such as print, direct mail, and broadcasting. As FSC recognized that more and more customers were searching online, their customer acquisition strategy changed to reflect the prevailing marketplace.

Today, FSC is taking a more critical approach to SEM in terms of strategy, discipline, and execution. SEM is composed of 30\% PPC and 70\% SEO. This suggests the increasing importance of working with professionals directly responsible for SEO strategy to ensure that FSC's strategy and synergies are horizontally and vertically integrated with the evolving marketplace.

One concern revolves around the rising bid costs for PPC keywords. FSC's main competitors control 70\% of the market, and they can have a dramatic effect on keyword prices that FSC with lower market share might bid on. Key financial terms like mutual funds and IRA are very expensive in terms of their bid price and therefore are highly-competitive. This has lead FSC to expand the number of managed keywords from 500 to more than 3,000. The FSC keyword list for PPC is managed through a high funnel theory, which directs users through a hierarchal approach based on primary keywords, which are very expensive. This strategy implies using a 'community of words' approach that uses secondary words that the largest competitors might not be bidding on. As a result the bid 
prices for these words are inexpensive, yet still useful in terms of driving traffic and results. The keyword bid function is a true art and expertise and that is why FSC outsourced this critical function to a third-party vendor. FSC now spends a lot more on PPC, having increased the budget by $160 \%$ over 2005 levels, allowing a PPC core and secondary markets. The core market includes such prime financial sites such as CNNMoney, SmartMoney, The Wall Street Journal, and Kiplinger's. The secondary market includes lesser known financial links.

The future of SEM at FSC is both exciting and challenging as they continue to build and grow organically its next generation of investors. Several things are monitored carefully:

- $\quad$ Google and Yahoo being efficient and effective as marketplace leaders

- $\quad$ Maintaining a top of mind status in terms of brand and loyalty

- $\quad$ Assessing new opportunities (e.g. investing IRS tax refunds)

- $\quad$ Validating customer sweet spot

- $\quad$ Driving lifetime value (6 to 7 years) through the initial sale as an indicator

From an execution perspective, FSC is focused on:

- $\quad$ Driving traffic through the core and secondary search engine sites by utilizing more than 3,000 keywords depending on the investor target audience.

- $\quad$ Continue building content that drives increased conversion rates and lifetime value.

- $\quad$ FSC is using new technology called expandable banners, which are ads that contain customized content that when opened direct this user to FSC's Website. So far, this new technology drives users, $75 \%$ of the time, deeper into the Website. This contributes to a higher conversion rate of customers.

- $\quad$ FSC emphasizes a common sense approach - timing is everything

\section{DISCUSSION}

Given the immergence of the Internet and the changing marketplace there is no singular answer to SEO or PPC, albeit careful consideration to a long term strategy consisting of both of these tactics to compliment one another may be the optimal solution in most business paradigms. It has been shown, especially within the industry interviews that one strategy will not work for everyone. Rather, the integration of these two practices as well as other Internet marketing tactics varies from business to business and rightly so. The synergy created by these two strategies certainly lends itself to benefits in terms of increased customer lifetime value, as well as, contribution to increased metrics, brand awareness and loyalty, and cultivating total customer experience. Additionally, it is important to note the role of generational marketing in terms of the push-pull dynamic and understanding how customers will engage your site along the pre-purchase decision continuum. At every step along that continuum is a "tipping point" to capture and influence consumer behavior towards the product while educating target audiences about the brand and value proposition. In the end, if marketers build an SEO solution with the education and service element in mind, that will ultimately lead to higher long term search engine results - perhaps not a number one ranking but certainly a Website that consistently ranks within the top ten. Never let a consultant assure you that your site's optimization is guaranteed a number one ranking through any search engine.

This does not mean that a site should swing all the way to the other side of the spectrum either. PPC is an invaluable tactic for large businesses, as well as, medium to small businesses. Having PPC paired with a naturally high ranking site is the optimal way to ensure that a site is not only noticed but visited as well. It is anticipated that the cost of utilizing PPC is going to soar even higher as more sites begin to jump on board. As the cost of PPC rises, entry barriers for smaller businesses will also begin to rise leaving them with one of two options: focusing more on naturally optimizing their site through the use of SEO or to focus more on investing in more words (secondary words) on PPC. As a strategy for middle to smaller sized businesses in the PPC world, a company must accept tradeoffs in regards to what words they intend to pursue versus others they may pursue with less conviction, because these firms are typically not the market leaders. 
Because of the lack of marketplace consensus, internet marketing strategies will continue to evolve and change. It remains imperative that in order to succeed in SEM strategies, one must develop an ever maturing long term or clock building approach. The fundamental fact remains that no business will ever figure out Google's or Yahoo!'s search engine algorithms; therefore, the key to success is a consistent, steady long term approach to drive corresponding high search engine results and consequently traffic. Lastly, while high keyword cost can be an obstacle in PPC, the longer term outlook is very optimistic as the appeal of search engine marketing will broaden accounting for a greater percentage of the business and marketing mix with SEO leading the way.

Aaker and Joachimsthaler (2000; 237) offer the following avenues for building brands on the Web: 1) advertising and sponsored content, 2) Intranet, 3) customer extranet, 3) Web PR, 4) e-mail, and 5) Web site. Aaker posits that a brand-building Website should consists of the following elements. First, a positive experience that is easy to use, delivers value, and is interactive, personalized, and timely. Second, it should reflect and support the brand. Third, one should look for synergy with other communication programs. Fourth, provide a home for the loyalist. And, lastly, one should differentiate with a strong subbranded content (Aaker and Joachimsthaler, 2000, p. 242). The key for marketing managers is to begin leveraging the brand by creating greater awareness or "top of mind" status amongst their consumers that shapes the total online experience and ultimately influences the purchase decision.

\section{REFERENCES}

1. Aaker, David A. and Erich Joachimsthaler. Brand Leadership. New York: Free Press, 2000.

2. Bannan, Karen J. Netmarketing: Strategies and Techniques for Web Marketers Organic Search Grows. $B$ to B. 90.2 (2005): 18-22. Business Source Premiere. 3 February 2007.

3. Bannan, Karen J. Strategies and Techniques for Web Marketers. B to B. 89.3 (2004): 18-22. Business Source Premiere. 3 February 2007.

4. Bloomberg, Toby. Internet Marketing Overview. American Marketing Association. 1 February 2007. $\langle$ http://www.marketingpower.com/content-printer-friendly.php\&item....

5. Boswell, Keith. Digital Marketing vs. Online Advertising. The Market Leap Report. 2.5 (2002): 1-3. 1 February 2007. <http://www.marketleap.com/report/ml_report_24.htm>.

6. Collins, James, and Jerry Pooras. Built To Last: Successful Habits of Visionary Companies. New York: HarperCollins Publishers Inc, 2002.

7. Guarnaccia, Tiffany. Use the Power of the Internet in Marketing Efforts. American Marketing Association. 1 February 2007. <http://www.marketingpower.com/content-printer-friendly.php?\&Item_ID=31874>.

8. Internet Advertising History. EC2 at USC. 13 March 2007. <http://www.ec2.edu/dccenter/archives/ia/history.html>.

9. Kerwin, Ann Marie and Leslie Taylor. Media Morph: Google Death Penalty. Advertising Age. 77.9 (2006): 51-51. Business Source Premiere. 3 February 2007.

10. Peters, Diane. SEO for CEOs. Profit. 24.5 (2005): 90-91. 3 February 2007. <http://Web.ebscohost.com/ehost/delivery?vid=13\&hid=16\&sid=3f06e763-58db- 47a3-8da2-a〉.

11. Raychaudhuri, Prabuddha S. Maximizing Your Paid Advertisement With Google. Search Engine Marketing Professional Organization. 1 February 2007. $\langle$ http://www.sempo.org/learning center/articles/adwords $>$.

12. Reichheld, Fred. The Ultimate Question: Driving Good Profits and True Growth. Bain \& Company, 2006.

13. Search Optimization Tactics to Avoid. B to B. 89.9 (2004): 19-19. 3 February 2007. $\langle$ http://Web.ebscohost.com/ehost/delivery?vid=23\&hid=16\&sid=3f06e763-58db-47a3-8da2-a ...>.

14. SEMPO, The State of Search Engine Marketing 2005. Search Engine Marketing Professional Organization. December 2005. 1 April 2007. 〈http://www.sempo.org>.

15. Shiva, V. A. The Internet Publishing Guide: How to Maximize Your Marketing and Promotion in Cyberspace. New York: Allworth Press, 1997.

16. Steele, Emily. 2007.Advertising's Brave New World Wall Street Journal, May 25, p B1+B3

17. Web Master Guidelines. Webmaster Help Center. 6 February 2007. 〈http://www.google.com/support/Webmasters/bin/answer.py?answer=35769>. 
18. Whalen, Jill. Search Engine Optimization and the Bottom-Line. SEMPO. 17 March 2007.

<http://www.sempo.org/learning center/articles/bottomline>.

\section{NOTES}

